\title{
PaclOOS Water Quality Sensor Partnership Program
}

By Shaun Wriston, Gordon Walker, Margaret Anne McManus, Simon Ellis, Fiona Langenberger, and Melissa Iwamoto

Ocean observing systems provide scientists, resource and conservation managers, industry, recreationists, and the general public with ocean data and information to improve decision-making (Iwamoto et al., 2016). While the need for improved open ocean data is global, data and information to address the needs of local, coastal communities is increasingly important and must be identified to effectively aid decision-making by local governing bodies, stakeholders, and those mentioned above.

Initiated as a pilot project in Honolulu in 2007, the Pacific Islands Ocean Observing System (PaclOOS; https:// www.pacioos.hawaii.edu/) was first certified in 2015 by the US Integrated Ocean Observing System (IOOS) as a Regional Information Coordination Entity. Its area of interest includes the State of Hawai'i; the territories of Guam, American Samoa, and the Commonwealth of Northern Mariana Islands; the Freely Associated States of the Federated States of Micronesia (FSM); the Republic of the Marshall Islands; the Republic of Palau; and the Minor Outlying Islands of Howland, Baker, Johnston, Jarvis, Kingman, Midway, Palmyra, and Wake. PaclOOS collects ocean data from partners across the region, develops and maintains numerical models and forecasts, and integrates this information into freely accessible data services and user-friendly web interfaces (Iwamoto et al., 2016).

PaclOOS also collaborates with various organizations and individuals who need water quality data on shorter timeframes to inform their work. The Water Quality Sensor

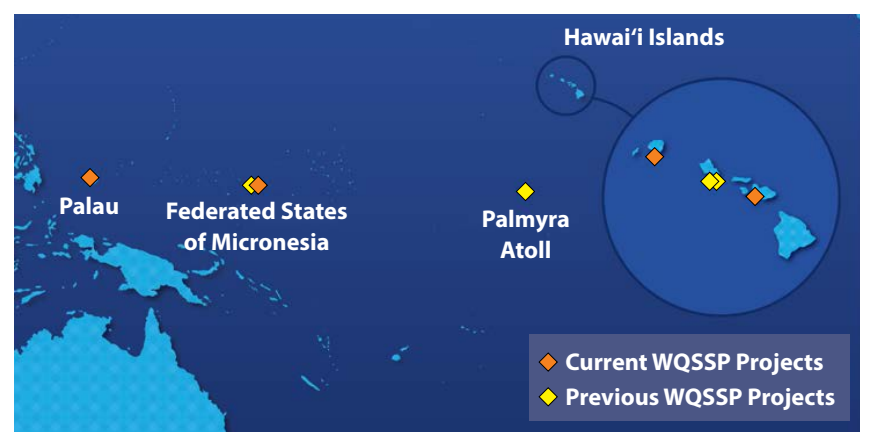

FIGURE 1. Map of current (orange) and previous (yellow) Pacific Islands Ocean Observing System (PaclOOS) Water Quality Sensor Partnership Program (WQSPP) projects. To date, partnership deployments extend throughout the PaclOOS region, including the Hawaiian Islands (Maui, O‘ahu, and Kaua'i); Palmyra Atoll; Pohnpei, Federated States of Micronesia; and Palau.

FIGURE 2. A diver maintains the water quality sensor at Kewalo Basin, O'ahu, which provided valuable data to the nonprofit organization Friends of Kewalos. Photo credit: PaclOOS
Partnership Program (WQSPP) supports scientists, natural resource managers, and citizens to collect data for research, conservation, planning, and resource management projects. Accurate and reliable oceanographic parameters are often difficult to obtain due to a lack of resources and/or technical expertise. The WQSPP aims to fill this gap by partnering with local project coordinators to increase the understanding of coastal marine ecosystems. State and government resource agencies, colleges, nongovernmental organizations, private businesses, and citizens, as well as independent researchers within the PaclOOS region can apply to use a Sea-Bird 16plus V2 SeaCAT water quality sensor for a period ranging from six months to two years. Sensor packages autonomously measure conductivity, temperature, and pressure with high precision, and up to seven auxiliary sensors can be simultaneously deployed to measure additional parameters such as chlorophyll and turbidity. In addition to the sensor suite, participants are provided with data management and technical capacity building assistance to allow for robust data collection.

An application for the WQSPP is initiated with a concept paper outlining the participant's location, eligibility, purpose, and impact; project duration; permitting requirements; management practices; and a map of the proposed field site. Successful applicants then work with PaclOOS staff to complete a more comprehensive application, and if selected, a project agreement plan. While PaclOOS supplies all the equipment, training, and data management, applicants are expected to pay for all costs associated with transporting and maintaining the sensor on site.

Presently, four sensors are being deployed in the WQSPP program (Figure 1). Data from these sensor packages are used to characterize temporal variability in the water column properties at each site. After data are collected, partners receive a summary document providing specific

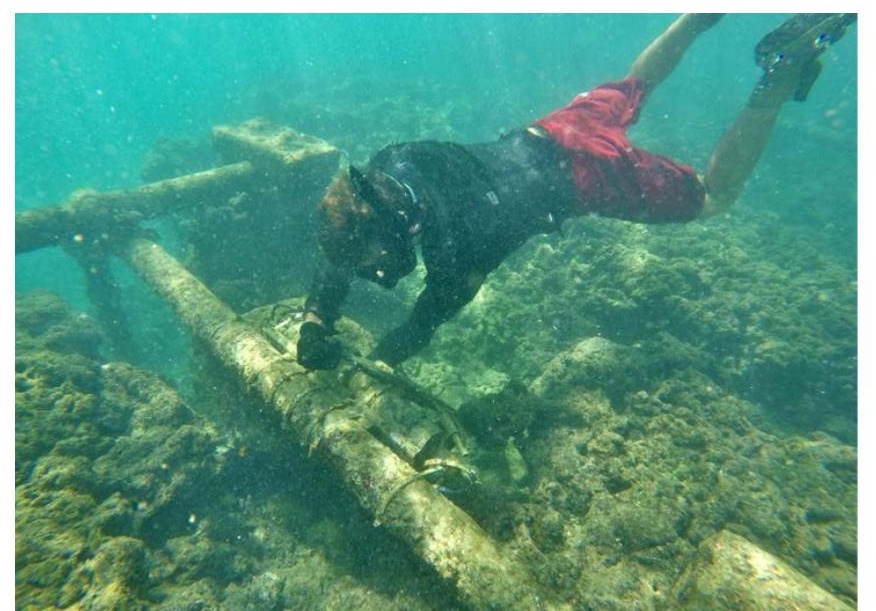




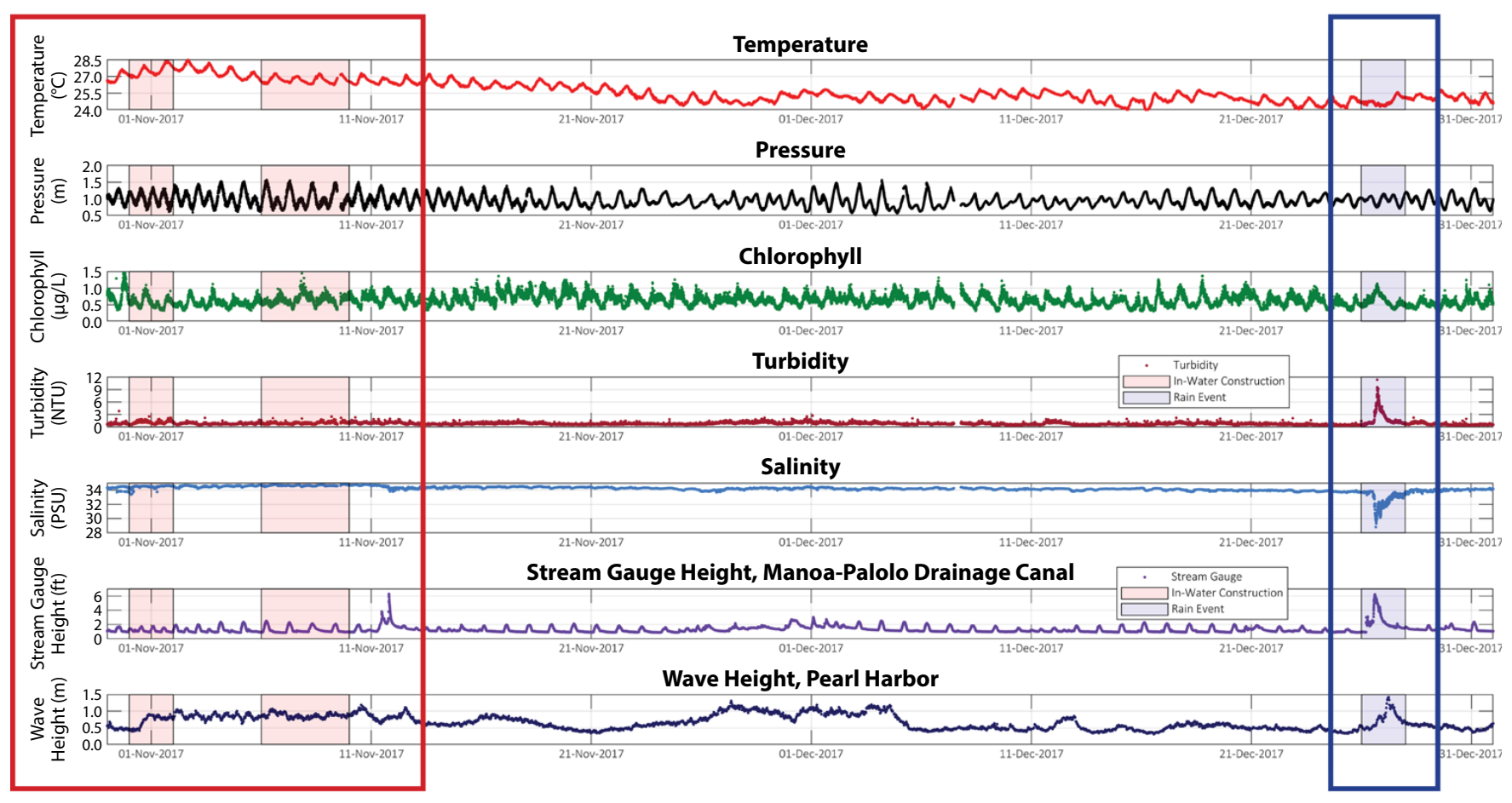

FIGURE 3. Data produced by PaclOOS WQSPP sensors for Friends of Kewalos. The figure focuses on in-water construction periods (red box) during the Kewalo Basin Harbor construction project and a rain event (blue box).

details of the project, visualizations, and descriptions of the data gathered by the sensor along with a case study addressing specific objectives of the deployment. Thus far, sensors at individual sites have provided insights into runoff patterns caused by heavy rainfall and progress in pollution cleanup, and they have clarified the impact from periods of in-water construction on local water quality.

One of the WQSPP projects was with Friends of Kewalos, based in Honolulu, Hawai'i. Friends of Kewalos worked with the PacIOOS WQSPP to deploy and manage a sensor located on the south shore of O'ahu in Māmala Bay, at Kewalo Basin (Figure 2). Friends of Kewalos is comprised of recreational users committed to protecting and preserving the Kewalo Basin Park and the surrounding shoreline and ocean. Their intent is to mālama (care for) Kewalo Basin, to ensure that its users will continue to have access to this site and the ability to enjoy it for generations to come.

Deployment for this partnership extended from September 2017 to May 2019, including a period of inwater construction at Kewalo Basin Harbor in October and November 2017. In September 2017, Kewalo Basin Harbor initiated an improvements project aimed at increasing harbor berth count, rehabilitating piers, and replacing a condemned loading dock and fueling system. This project included both out of water and in-water construction, and standard erosion barriers were put in place to prevent sediment from leaving the construction site. Friends of Kewalos and other community members were concerned that this construction (particularly in-water) may have adverse effects for both the surrounding marine environment and those who enjoy it. Measurements taken by the nearshore sensor did not show any significant changes in the parameters measured over the course of each in-water construction period.

A second result from this WQSPP deployment focused on a rain event that occurred on December 26, 2017. As the neighboring stream gauge height (US Geological Survey) rose to $6.21 \mathrm{ft}$, salinity levels dropped down to 28.81 practical salinity units, chlorophyll and turbidity levels rose, and temperature dropped to its lowest reading during deployment, $24.27^{\circ} \mathrm{C}$, showing how a single environmental event can affect many aspects of nearshore waters (Figure 3 ).

Through the collection of water quality information, partnerships like the PaclOOS WQSPP and Friends of Kewalos help natural resource managers and researchers to better evaluate their projects and make more informed decisions about them. PacIOOS aims to grow the number of sensor packages available and projects supported as interest and resources continue to grow.

PaclOOS would like to acknowledge and thank our partners at Maui Nui Marine Resource Council, Ebiil Society, Conservation Society of Pohnpei, Kaua'i Sea Farms, The Mariana Islands Nature Alliance, US Fish and Wildlife Service, Friends of Kewalos, and Mālama Maunalua.

\section{REFERENCE}

Iwamoto, M.M., F. Langenberger, and C.E. Ostrander. 2016. Ocean observing: Serving stakeholders in the Pacific Islands. Marine Technology Society Journal 50(3):47-54, https://doi.org/10.4031/MTSJ.50.3.2.

ARTICLE DOI: https://doi.org/10.5670/oceanog.2021.supplement.02-25 


\section{AUTHORS}

Shaun Wriston (swriston@hawaii.edu), Department of Oceanography, University of Hawai'i at Mānoa, USA. Gordon Walker, Pacific Islands Ocean Observing System, and Department of Oceanography, University of Hawai'i at Mānoa, USA. Margaret Anne McManus, Department of Oceanography, University of Hawai'i at Mānoa, USA. Simon Ellis, Pacific Islands Ocean Observing System, and University of Hawai'i Sea Grant, USA. Fiona Langenberger and Melissa Iwamoto, Pacific Islands Ocean Observing System, USA.

\section{ARTICLE CITATION}

Wriston, S., G. Walker, M.A. McManus, S. Ellis, F. Langenberger, and M. Iwamoto. 2021. PaclOOS Water Quality Sensor Partnership Program. Pp. 66-67 in Frontiers in Ocean Observing: Documenting Ecosystems, Understanding Environmental Changes, Forecasting Hazards. E.S. Kappel, S.K. Juniper, S. Seeyave, E. Smith, and M. Visbeck, eds, A Supplement to Oceanography 34(4), https://doi.org/10.5670/oceanog.2021. supplement.02-25.

\section{COPYRIGHT \& USAGE}

This is an open access article made available under the terms of the Creative Commons Attribution 4.0 International License (https://creativecommons.org/ licenses/by/4.0/), which permits use, sharing, adaptation, distribution, and reproduction in any medium or format as long as users cite the materials appropriately, provide a link to the Creative Commons license, and indicate the changes that were made to the original content. 